\title{
Research of Logical Diagnoses of Device Tripping Based on SCADA Steady Information
}

\author{
Lei Shi ${ }^{1, a}$, Yong Zhai ${ }^{2, b}$, Chenglong Feng ${ }^{2, c}$ and Zhenyu Ruan ${ }^{2, d}$ \\ ${ }^{1}$ Tianjin Electric Power Corporation Electric Dispatching and Communication Center, Tianjin, \\ 300010, China; \\ ${ }^{2}$ Beijing Kedong Electric Power Control System CO., LTD, Beijing, 100000, China. \\ alei.shi3@tj.sgcc.com.cn, bzhai_yong@163.com, '775998117@qq.com, d793801644@qq.com
}

Keywords: tripping alarm, accident handling, relay protection.

\begin{abstract}
Based on Tianjin $220 \mathrm{kV}$ power grid topology structure and protection setting configurations, the alarm steady information is mined deeply. Moreover, the big data of SCADA steady information is took full advantage to realize the accurate judges of all kinds of device tripping and the auxiliary information function of the reminding of relative protective action, which offers richer and more accurate alarm information and promotes the emergency handling ability for complex power grid.
\end{abstract}

\section{Introduction}

With the growing of the power grid scale and the increase of reliability requirements of urban power supply, operators should have the ability with higher levels of processing power grid accident. Currently, the integrated intelligent analysis and alarm applications of the D5000 scheduling technology support system has been realized the multi-alarm source comprehensive judgment with the grid fault tripping[1]. However, the determining logic for equipment tripping using SCADA steady-state data is too simple, and it is relative single for the applicable network topology and connection form[2]. Due to its mainly relying on the total signal of substation accident and switch judgment of remote communication, the false alarm information is leaded to when in the process of the equipment maintenance operation and the difficulties to effectively and accurately determine the more complex forms of tripping such as the protection failure caused by switch refusing action, the backup protection action at the next higher level and so on[3]. At the same time, for other alarm data source, they rely too much on the protection and wave record information that giving by the stand-side protection information collection and industrial computer, which is limited by the hardware conditions of the field erection and daily patrol maintenance frequency. Once the delay sending that lead by the freezes or not allowed of the Stand-side protection information collection and industrial computer, it will affect the alarm information judgment[4]. Therefore, in order to make full use of the multifocal data source of scheduling main-side and improve the accuracy and timeliness of equipment tripping alarms information, in this paper, the SCADA steady-state data is depth mined and its big data features and the advantages of high quality are employed, and based on Tianjin $220 \mathrm{kV}$ power grid topology and the plant diversity of connection form as well as combined with relevant protection configuration setting and behavior, the logic judgment suitable for the complex fault tripping is formed, prompting dispatcher the related protections for quickly and accurately locating of the fault point when handle accident.

The following parts conduct fault tripping judgment for the line fault, transformer faults, and bus fault aiming at the main equipment in Tianjin which is in $220 \mathrm{kV}$ topology, station configuration wiring and protection setting.

\section{Line fault tripping judgment}

According to the $220 \mathrm{kV}$ network topology and line protection actions, we consider as follows: 
1) L1 ring network circuitry, which is equipped with two sets of longitudinal current differential and backup protection. When detect both ends of L1 switch jumped at the same time (less than 2 seconds displacement time interval), due to the consideration of the remote devices between stations and the time difference caused by network transmission, if there is no information of bus bar differential protection on both ends of L1 or switch failure protection action on the bus within 2 seconds, prompt the alarm information as follows: L1 has fault, do the circuit protection (longitudinal, distance, zero flow) action.

2) The load line L2, namely the single belt line, of which the trip plate of circuit protection device on load-side opens. When detect the jumping of line switch of L2 on the power supply side, due to the consideration of the protect action of bus coupler/section switch in load side station after 2seconds, if L2's lateral load line switch always maintain close without mother bus bar differential protection on both ends of L2 or switch failure protection action information on the bus within 2 seconds, prompt the alarm information as follows: L2 has fault, do the circuit protection (longitudinal, distance, zero flow) action.

Due to the consideration of the adjusting of the power grid or the change of ring line into a single line temporarily caused by the line repair work, in order to enhance the intelligence of the judgment logic and reduce the daily artificial maintenance, when the displacement of one $220 \mathrm{kV}$ line switch happens, trigger the automatic scanning identification on the single line[5]. Specifically, investigate each $220 \mathrm{kV}$ line on the bus, if one can search $500 \mathrm{kV}$ transformer or generating set along the real-time network topology, it is seen as a power circuit on the bus. When and only when there is only one power supply circuit on the bus, the power supply circuit is the single circuit.

3) Empty filling line L3, which has only one side line switch in the running state. For the goal that there is no false alarm when doing the turning into inspection operation of line, when detect the jumping of unilateral line switch of L 3 and the total accident signal of corresponding stations occurs at the same time (the time interval is less than $0.3 \mathrm{~s}$ ), prompt the alarm information as follows: L3 has fault, do the line protection (longitudinal, distance, zero flow) action.

4) Wire is set Ti wire and transformer without high side by the general line of L4. When considering the turning into inspection operation of $\mathrm{L} 4$ or $\mathrm{Ti}$, it is needed that $\mathrm{Ti}$ is loaded backward and the neutral grounding switch of Ti on high side is closed first, and then switch of L4 on the power supply side can be opened. Therefore, when neutral grounding switch is tripping in Ti on high side, if the jumping of line switch of L4 on the power supply side is detected and there is neither fault tripping information of $\mathrm{Ti}$ nor the protected action information of bus differential protection or switch failure on the bus bar of L4 on the power supply side within 2 seconds, prompt the alarm information as follows: L4 has fault, do the line protection (longitudinal, distance, zero flow) action.

\section{Transformer fault tripping judgment}

As a general rule, the transformers are equipped with double set of longitudinal differential protection, non-electricity protection, which are seen as their main protection, and equipped with neutral point (gap and small resistance) as their backup protection. Let I and II period protections of after/zero flow of three sides of main switch as the backup protection of transformer, medium/low voltage bus or power distribution line, etc.

Set $220 \mathrm{kV} / 210 \mathrm{kV} / 35 \mathrm{kV}$ three-phase transformer of typical Tianjin $220 \mathrm{kV}$ substation as an example, which the wiring diagram is shown in Figure 1. 


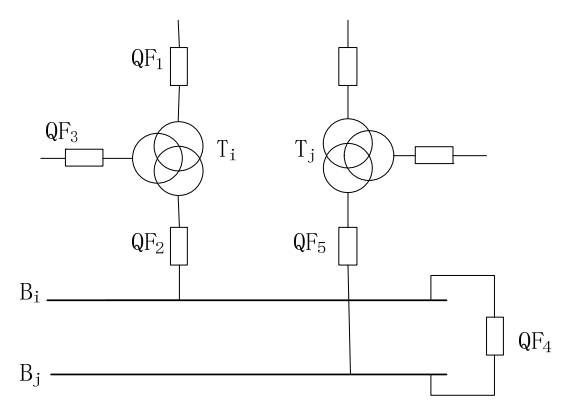

Fig. 1 Wiring diagram of $220 \mathrm{kV} / 110 \mathrm{kV} / 35 \mathrm{kV}$ transformer

Considering the turning into inspection operation of standing among/low voltage bus bar in the stations or transformers, and under the special circumstances that transformer fault tripping occurs after medium/low voltage total switch QF2 or QF3 opened, transformer fault tripping judgment logic is formed as shown in Table 1.

Table 1 Transformer fault judgment logic $(220 \mathrm{kV} / 110 \mathrm{kV} / 35 \mathrm{kV})$

\begin{tabular}{ll}
\hline \multicolumn{1}{c}{ Judgment logic } & \multicolumn{1}{c}{ Alarm information } \\
\hline 1.Detect QF1 and QF2 jumped at same time & "Ti has fault, transfer differential or Heavy Gas dose \\
(Displacement time interval is less than 0.3s). & protection action" \\
2.Detect QF1 and QF3 jumped at same time & "Ti has fault,QF1 reflow/zero flow or neutral pointdose \\
(Displacement time interval is less than 0.3s). & protection action" \\
3.QF2 and QF3always jumped and detect QF1 & "Ti lead of 110kV or neutral pointhas fault,QF2 zero \\
jumped and the total accident signal appeared & $\begin{array}{l}\text { flow section II dose protection action" } \\
\text { at the same time(Displacement time interval is }\end{array}$ \\
"Ti lead of 35kV or neutral pointhas fault,QF3 zero \\
less than 0.3s).
\end{tabular}

If the total switch refusing on one side happens when the transformer tripping, it will cause relative failure protection or backup protection, in which total switch refusing on high side can be referred to the logic judgment for breaker failure protection in Section 4.1, and the logic judgment for total switch refusing on medium/low voltage side (set QF2 for example) is shown in Table 2.

Table 2 Judgment logic of main refusing on medium/low voltage side when the transformer is tripping $(220 \mathrm{kV} / 110 \mathrm{kV} / 35 \mathrm{kV})$

\begin{tabular}{lc}
\hline \multicolumn{1}{c}{ Judgment logic } & \multicolumn{1}{c}{ Alarm information } \\
\hline 1.QF2 always closed,QF4 always jumped,QF1 和QF3 jumped at same time & "refused action of \\
(Displacement time interval is less than 0.3s) & switchQF2" \\
2.QF2 always closed,QF1 and QF3 at the same time jump (Displacement time & \\
interval is less than 0.3s) within 2 $4 \mathrm{~s}$ after QF5 also jumped(QF5backup & \\
protection action). & \\
3.QF2and QF4 always closed,QF5 always jumped,QF1和QF3 jumped at same & \\
time (Displacement time interval is less than 0.3s) & \\
4.QF2and QF4 always closed,QF1 and QF3 at the same time jump \\
(Displacement time interval is less than 0.3s) within 2 4 s after QF5 also \\
jumped(QF5backup protection action).
\end{tabular}

Among them, the general reflow I period protection direction of QF2 points to transformer Ti, the reflow protection of QF3 is without a direction, therefore, they are also as backup protection of Ti fault. Set the reflow I period protection of QF2 as example, if Ti has fault, only when its two sets differential protection and the heavy gas protection have not been action, and there is little power (place, owned unit grid connection line) on bus Bi or there is other power supply ( $\mathrm{Tj}$ or little power) on the bus $\mathrm{Bj}$ that is parallel with $\mathrm{Ti}$, it will jump size side by the main switch by the complex flow I period action of QF2. Therefore, such protection action has extremely low probability, and it is not listed in Table 2.

For the other of $220 \mathrm{kV} / 210 \mathrm{kV} / 35 \mathrm{kV}$ three-phase transformers can refer to the above analysis and logical design. 


\section{Bus fault tripping judgment}

\section{$4.1220 \mathrm{kV}$ bus fault}

Consider the following situations respectively according to the $220 \mathrm{kV}$ bus connection form.

1) The appose/parse connection form of double bus and the segment connecting form of single bus, which are as shown in Figure 2(a) and Figure 2(b). Bus is equipped with two sets of protective device (generally contains bus differential, breaker failure, bus connection being failure, bus connection dead zone and bus connection charging protection) and bus connection switch has auto-reclosing protection. The logic judgment conditions according to the protection action are shown in Table 7.
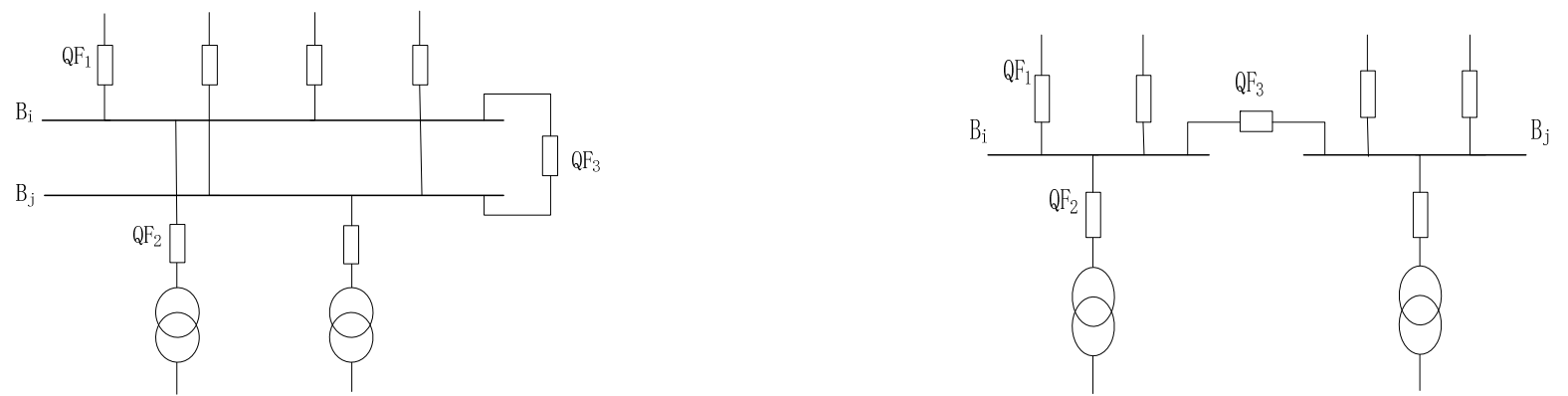

Fig. 2(a) 220kV appose/parse connection form of double bus Fig. 2(b) $220 \mathrm{kV}$ segment connection form of single bus

Table 3 Judgment logic of bus protection device action of $220 \mathrm{kV}$ single/double bus connection

Judgment logic

1.When detect all closed switches (line switch, transformer master switch and bus connection/segment switch) that connected to Bi are jumped at the same time (displacement time interval is less than $0.3 \mathrm{~s}$ ).

2. When detect all closed switches that direct connected to Bi are all jumped at the same time excepted a line switch QF1 ( excepted the load side switch of single line) or master switch QF2 of transformer on high voltage side, all other closed switches that direct connected to it are jumped at the same time (bus connection/segment switch of failure protection jumped at Itime limit, master switch of bus line and transformer high voltage side jumped at II time limit, displacement time interval is less than $0.5 \mathrm{~s}$ ).

3.Except QF3 always stay closed, when detect all closed switches that direct connected to aralleling running bus $\mathrm{Bi}$ and $\mathrm{Bj}$ jumped at the same time (after bus differential protection of bus Bi jumped, bus connection failure protection $\mathrm{Bj}$ jumped with delay, displacement time interval is less than $0.5 \mathrm{~s}$ ) 4.If $\mathrm{Bi}$ and $\mathrm{Bj}$ are arallelrunning, when detect that all closed switches that direct connected to $\mathrm{Bi}$ and $\mathrm{Bj}$ are jumped at the same time (after bus differential protection of bus Bi jumped, bus connection dead zone protection $\mathrm{Bj}$ jumped, displacement time interval is less than $0.5 \mathrm{~s}$ )

5.If bus $\mathrm{Bi}$ and $\mathrm{Bj}$ are parse running, when detect that all closed switches that direct connected to $\mathrm{Bi}$ are jumped at the same time (displacement time interval is less than $0.3 \mathrm{~s}$ ).

6.All switched that direct connected to Bi are jumped, when detect QF3 turning from open to closed, jumped it within $0.5 \mathrm{~s}$.

7.When detect line switch of all power line on Bi are closed, QF3 turns from open to closed within $5 \mathrm{~s}$.

2) Bus in the form of internal bridge connection is within the protection scope of in-station transformer, therefore, there is no bus differential protection. The logic judgment of segment switch having auto-reclosing protection action can be referred in 7 in Table 3.

3 ) Bus in the form of $3 / 2$ connection, which is shown in Figure 3. Bus differential is equipped with double protection; in addition, there are protection of short approach lines and the failure
"Bi has failure, do the bus differential protection action "

"QF1/QF2 switch refused, breaker failure protection action "

"QF3 switch refused, bus connection failure protection action"

" section between QF3 and

CT has failure, bus connection/segment switch dead zone protection action"

"Bi has failure, QF3 charging protection action " "QF3 has auto action" 
protection of the protective equipment integrated in breaker. According to the above protection actions, judging logic of devices tripping is given in Table 4.

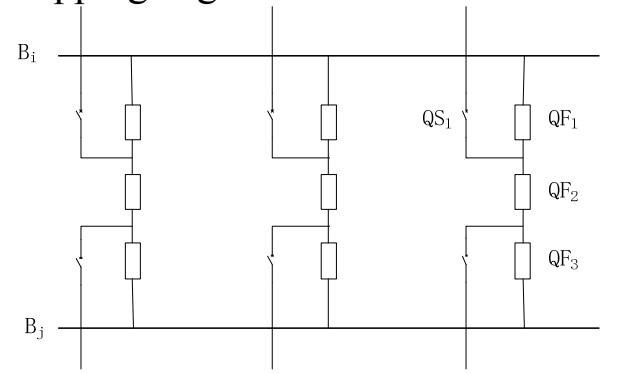

Fig. $3220 \mathrm{kV}$ bus $3 / 2$ connection form

Table 4 Judgment logic of 220kV3/2 connection bus differential, switch failure and short approach lines protection action

\begin{tabular}{|c|c|}
\hline Judgment logic & Alarm information \\
\hline $\begin{array}{l}\text { 1.When detect all closed switches connected with bus Bi are jumped at } \\
\text { same time (displacement time interval is less than } 0.3 \mathrm{~s} \text { ) }\end{array}$ & $\begin{array}{l}\text { "Bi has failure, do the bus } \\
\text { differential protection action }\end{array}$ \\
\hline $\begin{array}{l}\text { 2.Except the switch QF1 that direct connected to bus Bi is always closed, } \\
\text { when detect breaker in middle QF2 that direct connected to QF1 jumped } \\
\text { within o.5s, other closed switches that direct connected to bus Bi are } \\
\text { jumped at the same time (displacement time interval is less than } 0.3 \mathrm{~s} \text { ) }\end{array}$ & $\begin{array}{l}\text { "QF1 has switch refused, the } \\
\text { breaker failure protection } \\
\text { action" }\end{array}$ \\
\hline $\begin{array}{l}\text { 3. Switch QF2 always closed, and the two sides switch connected with QF2 } \\
\text { jumped at same time (displacement time interval is less than } 0.5 \mathrm{~s} \text { ). }\end{array}$ & $\begin{array}{l}\text { "QF2 has switch refused, the } \\
\text { breaker failure protection } \\
\text { action" }\end{array}$ \\
\hline $\begin{array}{l}\text { 4. When the } 220 \mathrm{kV} \text { line or the QS1 switch of the transformer open, switch } \\
\text { QF1 and breaker in middleQF2 are jumped at the same time in the stations } \\
\text { (displacement time interval is less than } 0.3 \mathrm{~s} \text { ). }\end{array}$ & $\begin{array}{l}\text { "Line between QF1 switch } \\
\text { and QF2 switch has failure, } \\
\text { short approach } \\
\text { linesprotection action" }\end{array}$ \\
\hline
\end{tabular}

\subsection{Medium/low voltage bus failure in stations}

Generally $110 \mathrm{kV}$ bus in $220 \mathrm{kV}$ station adopts the appose/parse connection form of double bus, as shown in Figure 4(a). Bus equipped with single set of protection device (generally contains bus differential, bus connection failure and bus connection charging protection) and bus connection switch has auto-reclosing protection. The judgment logic of $110 \mathrm{kV}$ bus failure and bus connection having auto-reclosing protection action can be reference to corresponding described in Section 4.1.
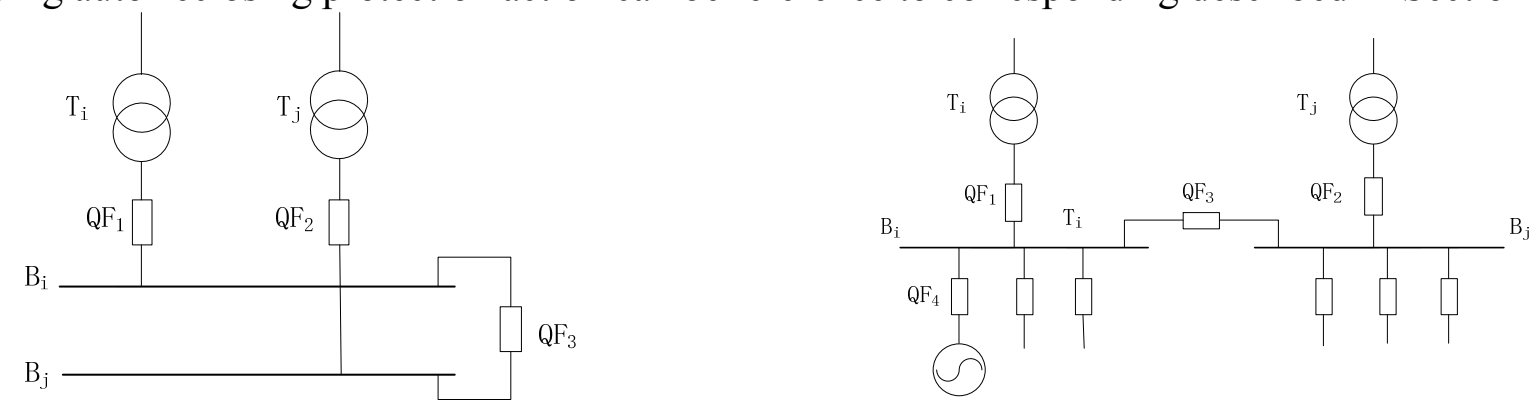

Fig. 4(a) $110 \mathrm{kV}$ appose/parse connection form of double bus Fig. 4(b) $35 \mathrm{kV}$ and10kV segment connection form of single bus

Generally $35 \mathrm{kV}$ and $10 \mathrm{kV}$ bus in $220 \mathrm{kV}$ station adopts the segment connecting form of single bus, as shown in Figure 4(b). Bus equipped with simple bus differential protection or no bus protection. The judgment of segment switch's auto-reclosing protection and charging protection can be reference to corresponding described in Section 4.1. Aim at low voltage bus failure, the judgment logic of simple bus differential protection action is shown in Table 5.

Table 5 Judgment logic of simple differential bus protection action of low-voltage bus failure

\begin{tabular}{ll}
\hline \multicolumn{1}{c}{ Judgment logic } & Alarm information \\
\hline 1.QF3 always jumped, active power telemetering value of QF1 is greater & "Bi has fault, simple \\
than0.1MW, when detect QF1 and small power source line switchjumped at the & $\begin{array}{l}\text { bus differential } \\
\text { same time (displacement time interval is less than 0.3s). }\end{array}$ \\
\hline
\end{tabular}


2.Active power telemetering value of QF1 is greater than $0.1 \mathrm{MW}$, when detect

(closing auto-cast)"

QF1、QF3 and QF4 jumped at the same time (QF3 protect the I time to jump,

$\mathrm{QF} 1 / \mathrm{QF} 4$ protect the II time to jump, displacement time interval is less than

$0.3 \mathrm{~s})$.

3.QF1 always jumped, QF2 always closed, active power telemetering value of

QF3 is greater than $0.1 \mathrm{MW}$, when detect QF3 jumped.

In addition, the reflow section II and zero flow zero-current section protection of the transformer on $110 \mathrm{kV}$ side master switch, the quick-break of master switch on $35 \mathrm{kV}$ side, reflow and zero flow section I protection, as well as the quick-break, reflow and z-type transformer zero flow protection, are also to be the backup protection of the connected bus failure or the switch refusing of power distribution line. It is important to note that, there exist some differences of the current $220 \mathrm{kV}$ configuration and setting in stations in Tianjin. In particular, the quick-break protection action on $35 \mathrm{kV}$ and $10 \mathrm{kV}$ side will lead to atresiaauto-plough. Due to the difference of protection setting principle for different construction period or the limitation of the field connection conditions, whether it will has atresiaauto-plough after protection action gets inconsistent. At the same time, whether the above protection action will jump bus connection or segment switch is also different. Therefore, jumped earlier before considering the bus coupler/section switch may cause another bus after neutral point grounding way change, so to protect the above configuration not jumped bus coupler/section switch function; However, for the need of improving power supply reliability and protection sensitivity, we in the set up the function of jumping bus connection or segment switch in first time limit. With the comprehensive consideration of the above protection actions, we show the judgment logic of switch tripping when medium/low voltage bus has failure or distribution outline switch refuses in Tables 6 and 7.

Table 6 Judgment logic of transformer master backup protection action when $110 \mathrm{kV}$ bus has failure

Judgment logic Alarm information

1.QF3 always jumped, active power telemetering value of

$\mathrm{QF} 1$ is greater than $0.3 \mathrm{MW}$, when detect QF1jumped and Bi

has not jumped of distribution line switch within $0.5 \mathrm{~s}$.

2.QF3 always closed, QF2 always jumped, active power telemetering value of QF1 is greater than $0.3 \mathrm{MW}$, when detect

QF1jumped and Bi has not jumped of distribution line switch within $0.5 \mathrm{~s}$.

3.when detect QF1 and QF3 jumped, Bi has not jumped of

distribution line switch within $0.5 \mathrm{~s}$.

4.QF1 always jumped, QF2 always closed, active power telemetering value of QF3 is greater than0.3MW, when detect QF3 jumped and ,Bi has not jumped of distribution line switch within $0.5 \mathrm{~s}$.

"Bi has distribution out line failure, line switch refused, QF1 reflow section II( or zero flow I section) action";

Distribution out line of Bihas fault

"Bi has failure, bus differential protection refused, QF1 reflow II section ( or zero flow section I) action"

5.QF3 always closed,QF1 and QF2 a jumped at the same time(displacement time interval is less than $0.3 \mathrm{~s}$ ), $\mathrm{Bi}$ has not jumped of distribution line switch within $0.5 \mathrm{~s}$.

\author{
"Bi or Bj has distribution out line failure, \\ line switch reused, QF1 and QF2 reflow \\ section II action"; \\ "Bi or Bj has failure, bus differential \\ protection refused, QF1 and QF2 reflow \\ section II action"
"Bi or $\mathrm{Bj}$ has distribution out line failure,
"Bi or Bj has failure, bus differential protection refused, QF1 zero flow section I action" \\ line switch refused, QF1 zero flow I section \\ action";
}

6.QF3 always closed, when detect QF1jumped and QF2 jumped within $1 \sim 2 \mathrm{~s}$.

Table 7 Judgment logic of transformer master backup protection action when $35 \mathrm{kV} / 10 \mathrm{kV}$ bus has failure

\begin{tabular}{cc}
\hline Judgment logic & Alarm information \\
\hline 1.QF3 always jumped, active power telemetering value of QF1 is & "Distribution of Bi has fault, line \\
\hline
\end{tabular}


greater than0.1MW, when detect QF1jumped.

2.Active power telemetering value of $\mathrm{QF} 1$ is greater than $0.1 \mathrm{MW}$, when detect QF3 and QF1 jumpedat the same time(QF3 jumped in I time limit of protection, QF1 jumped in II time limit of protection, displacement time interval is less than $1.3 \mathrm{~s}$ ) .

3.QF1 always jumped, QF2 always closed, active power telemetering value of QF3 is greater than $0.1 \mathrm{MW}$, when detect QF3 jumped QF1. 4.QF3 always closed,QF2 always jumped, active power telemetering value of QF1 is greater than $0.3 \mathrm{MW}$, when detect QF1 jumped. switch refusing action ,

QF1protection actionof quick-break and over-current or zero-current

"Bi has fault, bus differential protectionrefusing action or not have bus differential protection, QF1protection actionof quick-break and over-current or zero-currentaction"

In Table 6 and 7, if the neutral point of transformer is directly grounding or without the small resistance grounding, the alarms do not contain zero flow action information. Moreover, in the conditions 5 and 6 in Table 6 , with the consideration of the parallel running of $110 \mathrm{kV}$ bus in $220 \mathrm{kV}$ stations, usually there is only $110 \mathrm{kV}$ neutral point of one transformer $\mathrm{Ti}$ is grounding directly. Therefore, when the $110 \mathrm{kV}$ bus is grounding and the bus differential protection is refusing, or its distribution out line is grounding but the line switch is refusing, if QF1 zero flow section I protection dose not set the function of jumping QF3, there is only QF1 has zero sequence current and let the zero flow section I protection action jump, while the QF2 zero flow section I of another transformer $\mathrm{Tj}$ has no action, which can only rely on its gap protection on $110 \mathrm{kV}$ side, that is, remove the three sides master switch of transformer after $1 \sim 2$ sdelay after QF1's action.

When test the maintenance operation of the transformers that in stations or the medium/low voltage bus, generally, the medium/low voltage should be loop closed at first, or let the relative local scheduling removes the all load when it adopts the form of single bus connection. In the concrete process of operation, we both consider the simultaneity of the two switch's turning from closed to open on the above judgment conditions, and we also consider the circumstance that there remain flow (load) before the jumping for the master switch or bus connection segment switch. Therefore, it can satisfy the requirements that there is no false tripping when in the process of equipment maintenance.

When switch displacement occurs, start the failure jumping judgment to relative equipment according to the above logic conditions. If there is only one switch action in the judgment logic, the judging process of the equipment failure may be temporarily latches and maintain 10s. If the switch should be failure refusing action by other logical judgment, it should be seen as the turning from closed to open, and being satisfied the previously latched judgment logic condition and pushed the corresponding equipment failure alarm information, so as to realize the function of remained accurate location the root fault equipment when switch refused.

\section{Summary}

Based on the SCADA steady-state information, we analyze the fault trip in different categories and with progressive layers for the $220 \mathrm{kV}$ topology, the lines connecting form in stations and the protection setting and equipping in the equipments in main power grid in Tianjin, forming the judgment logic that adapted to the complex fault trip, and thus helping the dispatcher locate the fault point accurately and quickly. The main conclusions are as follows.

1) The fault analysis of the equipment trip needs to be conducted in different categories. For the fault analysis of equipment trip that similar to the $220 \mathrm{kV}$ power grid in Tianjin, the analysis should be analyzed in such three categories that line fault, transformer fault and bus fault.

2) The fault analysis of the equipment trip in each category, it should be progressive in hierarchical. For the line fault analysis, the progressive analysis should be in four layers, namely the loop network line,loading line,charging line and variable resistance line connection $\&$ high voltage side of transformer without main switch line. For the transformer fault analysis, the progressive analysis should be in three layers, namely $220 \mathrm{kV} / 110 \mathrm{kV} / 35 \mathrm{kV}$ three-phase transformer, $220 \mathrm{kV} / 110 \mathrm{kV} / 35 \mathrm{kV}$ inner bridging transformer and $220 \mathrm{kV} / 35 \mathrm{kV} / 10 \mathrm{kV}$ three-phase transformer. For the bus fault analysis, the progressive analysis should be in two layers, namely $220 \mathrm{kV}$ bus fault and medium/low voltage bus fault, and by four forms, namely the appose/parse connection form of 
double bus, the segment connection form of single bus, the form bridging of inner connection and the form of $3 / 2$ connection.

3) The fault analysis of the equipment trip needs to be conducted combining with switch refusing. If apply the trip fault analysis results and there is only one switch action after starting the corresponding equipment, the judgment progress of the equipment fault must be locked for 10 seconds. During this time, if the switch is judged to be failure refusing by other logic, the corresponding equipment fault alarm information should be pushed, thus to realize the purpose of accurate location even the switch is refusing.

4) The fault analysis of equipment trip needs to be conducted in a dynamic think. For each power grid, it has its own unique quality. Therefore, when the equipment trip fault happens, it should be combined with the characteristics of the power grid and employs the analysis principle and method that conducting with categories, with hierarchical progressive and with the switch refusing in a flexible way. In this paper, the fault analysis results of equipment trip is formed based on $220 \mathrm{kV}$ power grid in Tianjin, which can be referred to other fields.

\section{Reference}

[1]. SHAN Xin, DAI Zemei, ZHANG Zhe et al. Research on and Application of Integrated Smart Alarm Based on Smart Grid Dispatching and Control Systems. Automation of Electric Power Systems, 2015,39(01):65-75.

[2]. HOU Fangdi, XIE Yuzhe, GU Wei et al. Application of Comprehensive Grid Intelligent Alarm Technology Based on Dispatching and Control Integration. Zhejiang Electric Power. 2013(12):70-72.

[3]. ZHAO Jiaqing, TANG Sheng, DING Hongen et al. Multi-suBject Intelligent Integrated Alarm Technology. Power Sysem Protection and Control, 2015(10):70-72.

[4]. NING Guoli, FANG Yanan, XING Ligong et al. Improvement of Zero-sequence Over-current Protection for Grounding Transformer in Low-resistance Grounding Power System[J]. Electric Power Automation Equipment, 2011,31(05):141-143.

[5]. WANG Jianzhong. 35kV Simple Bus Protection Action Analysis . Automation of Electric Power Systems, ,2006,30(14):105-107. 